\title{
The Effect of Age, Postoperative Refraction, and Pre- and Postoperative Pupil Size on Halo Size and Intensity in Eyes Implanted with a Trifocal or Extended Depth-of-Focus Lens
}

\author{
Takeshi Teshigawara (1D ${ }^{1-3}$ \\ Akira Meguro $\mathbb{1}^{3}$ \\ Nobuhisa Mizuki ${ }^{3}$ \\ 'Department of Ophthalmology, \\ Yokosuka Chuoh Eye Clinic, Yokosuka, \\ Kanagawa, 238-0008, Japan; ${ }^{2}$ Tsurumi \\ Chuoh Eye Clinic, Yokohama, Kanagawa, \\ 230-005I, Japan; ${ }^{3}$ Department of \\ Ophthalmology, Yokohama City \\ University School of Medicine, \\ Yokohama, Kanagawa, 236-0004, Japan
}

\begin{abstract}
Purpose: Halos are a chief source of patient dissatisfaction after multifocal intraocular lens (IOL) implantation. The primary purpose of this study was to investigate the influence of age, postoperative refraction, and pre- and postoperative pupil size on postoperative halo size with a trifocal diffractive IOL (AcrySof IQ PanOptix) and extended depth-of-focus (EDOF) IOL (TECNIS Symfony) to determine whether these factors could predict postoperative halo size.
\end{abstract}

Patients and Methods: This single-center, open-label study conducted between October 2018 and April 2020 in Yokosuka, Japan included 160 patients: 80 with PanOptix and 80 with Symfony IOLs. Size and intensity of the halos were examined binocularly using a computer-based simulator (Eyeland Design Network $\mathrm{GmbH}$ ) and scored from 0 (minimum) to 10 (maximum) at 1,3 , and 6 months postoperatively, along with the change in each variable for both IOLs. The respective correlations of halo size with age, postoperative refraction, pre- and postoperative pupil size, and discomfort level due to the halo were also investigated 6 months postoperatively.

Results: Halo size and intensity were significantly mitigated between 1, 3, and 6 months postoperatively. Throughout the observation period, halo size was significantly larger with PanOptix than with Symphony $(\mathrm{P}<0.05)$, and halo intensity was significantly higher with Symphony than with PanOptix $(\mathrm{P}<0.05)$. The pre- and postoperative pupil size correlated positively with halo size, while age and postoperative refraction were negatively correlated, for both IOLs.

Conclusion: Understanding the features and predictive preoperative factors of halos in different types of multifocal IOLs may be useful in improving patient satisfaction.

Keywords: halo size, multifocal intraocular lens, biometric predictable factors, trifocal intraocular lens, extended depth-of-focus intraocular lens, photic phenomena, halo

\section{Introduction}

Patient satisfaction does not always differ between monofocal and multifocal intraocular lenses (IOLs), despite the fact that multifocal IOLs provide better near visual acuity and greater spectacle independence than do monofocal IOLs. ${ }^{1,2}$ Photic phenomena, such as halo and glare, are among the chief sources of patient dissatisfaction after multifocal IOL implantation. ${ }^{3}$ In 2018, Mencucci et $\mathrm{al}^{4}$ investigated a total of 120 eyes in 60 patients with multifocal IOLs using Symfony (40 eyes) (Abbott Medical Optics), PanOptix IQ (40 eyes) (Alcon), and
Correspondence: Takeshi Teshigawara Department of Ophthalmology, Yokosuka Chuoh Eye Clinic, 2-6 Odaki-cho, Yokosuka, Kanagawa, 238-0008, Japan Tel +8I 46827400 I

Fax +8I 468274141

Email teshitake@gmail.com 
AT LISA tri 839MP (40 eyes) (Carl Zeiss Meditec) and reported that $70 \%$ and $50 \%$ of patients with multifocal IOLs experienced halo and glare, respectively; moreover, halo was the most severe and aggravating postoperative optical phenomenon with each IOL. ${ }^{4}$ Previous studies have shown that a decrease in contrast sensitivity, followed by a photic phenomenon, were the most common causes of IOL explantation after multifocal IOL implantation. ${ }^{5,6}$ Zhao et al $^{1}$ noted a significantly higher incidence of halos in patients with multifocal IOLs than in those with monofocal IOLs. A variable degree of photic phenomena was also noted with multifocal IOLs. RuizMesa et $\mathrm{al}^{7}$ concluded that the differences between the photic phenomena for the commonly used multifocal IOLs, such as PanOptix and Symfony, were not significant. On the contrary, Skiadaresi et $\mathrm{al}^{8}$ indicated greater halo intensity with the Symfony than with the PanOptix IOL. Hence, the occurrence and intensity of photic phenomena with various IOLs require further research. One of the objectives of this study was to compare the size and intensity of halos in patients implanted with the PanOptix and Symfony IOLs. We also investigated and compared the change in the halo size and intensity with both IOLs 1 , 3 , and 6 months postoperatively since a previous study indicated that the photic phenomena are mitigated in the postoperative period. ${ }^{6}$

Several studies have shown how photic phenomena originate from the design of the IOL, such as apodization or non-apodization, sphericity or asphericity, degree of additional power, and lens-edge design. ${ }^{9-11}$ From the perspective of optics, diffractive multifocal IOLs are designed to distribute light to different foci and increase spectacle independence among patients. ${ }^{11}$ This theory indicates that the image is always overlaid by 1 secondary out-of-focus image in bifocal IOLs or 2 secondary out-of-focus images in trifocal IOLs, derived from the additional foci of the IOLs, which in turn induces the photic phenomena. ${ }^{12}$

The degree of halo was also attributable to some biometric variables such as kappa angle and pupil center shift, in addition to the IOL design and optical theory. ${ }^{13}$

However, there is a scarcity of studies focusing on factors for the prediction of halo size. ${ }^{14-16}$ Therefore, we also analyzed age, postoperative refraction, and pre- and postoperative pupil size as potential predictors of halo size in multifocal IOLs. Moreover, the level of patient discomfort due to the halo was investigated.

Given the fact that photic phenomena are among the most common factors causing dissatisfaction with the use of multifocal IOLs (especially halos, which cause substantial annoyance to patients), in-depth familiarity with the difference between the degree of halo for various multifocal IOLs and predictors of halo size may be vital in informing patients about the postoperative halo before surgery. Furthermore, the findings of this study may be helpful to ophthalmologists in providing their patients with personalized information regarding the prediction of severity of the halo, depending on the type of multifocal IOL and other factors. This was the principal aim of this study.

\section{Materials and Methods}

This single-center, open-label study was conducted at Yokosuka Chuo Eye Clinic. All physical examinations, surgical procedures, and research were performed from October 2018 to September 2020. Informed consent was obtained from all patients after explaining the study protocol in detail. All research procedures were conducted in accordance with the Declaration of Helsinki. The study was approved by the ethics committee of Yokosuka Chuo Eye Clinic.

\section{Patient and IOL Selection}

All patients enrolled in this study visited the clinic because of worsening vision quality and were diagnosed with cataracts. Other inclusion criteria included the following: a best-corrected distance visual acuity under $20 / 20$ or a lower than normal result on contrast sensitivity testing (Contrast Glare Tester CGT-1000, Takagi, Japan). Patients with other visual or systemic disorders affecting vision, such as diabetic retinopathy, glaucoma, age-related macular degeneration, uveitis, or a history of intraocular surgery, were excluded to eliminate possible confounding factors that could influence the outcomes. Ophthalmologists explained the design of each IOL, and the patient selected which IOL to receive, PanOptix (Alcon, Fort Worth, TX, USA) or Symfony (Abbott Medical Optics/AMO, Inc., Santa Ana, California, USA, now Johnson-Johnson Vision).

The AcrySof IQ PanOptix Model TFN00 IOL, launched in 2015, is an ultraviolet (UV), blue-light filtering, and non-apodized, diffractive, foldable, presbyopiacorrecting IOL with a refractive index of 1.55. This singleunit IOL with 2 open C-loop haptics is composed of a hydrophobic acrylic, an acrylate/methacrylate copolymer, with a central biconvex optic lens, as well as an inner diffractive and outer refractive zone. The total length of the IOL is $13 \mathrm{~mm}$, and its optic diameter is $6 \mathrm{~mm}$. The 
anterior part of the lens is spherical, and the posterior part is aspherical. The central part of the optical zone, measuring $4.5 \mathrm{~mm}$, is a diffractive surface with an additional power of 2.17 diopters (D) for intermediate $(60 \mathrm{~cm})$ and $3.25 \mathrm{D}$ for near $(40 \mathrm{~cm})$ vision, while $50 \%$ of light energy is allocated for distance vision and $25 \%$ each for intermediate and near vision.

This diffractive structure allows $88 \%$ of light to pass through a pupil size of $3.0 \mathrm{~mm}$, and optimizes performance in various lighting conditions, due to lesser dependence on pupil size. ${ }^{17,18}$

The TECNIS Symfony IOL, launched in 2014, a single-unit IOL with 2 open C-loop haptics, is a biconvex, UV-blocking, hydrophobic, acrylic extended depth-of-focus (EDOF) IOL with a refractive index of 1.47. This EDOF lens has $1.75 \mathrm{D}$ additional power for intermediate $(70 \mathrm{~cm})$ vision. The TECNIS Symfony has a proprietary achromatic diffractive surface and echelette design that offers low-add foci, which extend the range of vision for intermediate through far distances. This unique lens surface design also corrects chromatic aberrations, which enhances contrast sensitivity. ${ }^{19}$

\section{Pre- and Postoperative Examinations}

All patients underwent a complete preoperative ophthalmic examination. Nuclear grading was performed according to the Emery-Little classification ${ }^{20}$ under slit-lamp examination by 5 experienced ophthalmologists. Vision testing for subjective refraction was performed by the same technician. Anterior segment swept-source optical coherence tomography was performed using the CASIA 2 (Tomey Corp, Nagoya, Japan) to measure the preoperative photopic pupil diameter. The postoperative target refraction was based on the patients' requests. For most patients, the target refraction was emmetropia; however, some patients with preoperative myopia preferred to maintain very slight myopia to see near objects more clearly postoperatively. In such cases, very slight myopia (from $-0.25 \mathrm{D}$ to $-0.5 \mathrm{D}$ ) was the target, even if it would slightly compromise some quality of vision.

The recommended IOL power and predicted postoperative refraction were calculated using the IOL Master 700 (Carl Zeiss, Oberkochen, Germany).

Complete ophthalmic examinations, including subjective refraction, were conducted 1, 3, and 6 months postoperatively. A computer-based simulator software (Halo \& Glare Simulator, Eyeland Design Network
$\mathrm{GmbH}$, Vreden, Germany) was used to evaluate the size and intensity of the halos without refractive correction at 1 and 3 months, and with and without refractive correction at 6 months postoperatively. Patients selected the type of halo they experienced (T1: diffuse halo ring, T2: starburst type, T3: distinct halo ring) and adjusted its size and intensity on a slide bar with a simultaneous visual representation on the screen. The size and intensity of the halo were scored from 0 (minimum) to 10 (maximum) based on the position of the slide bar (there is no unit for these scores). Postoperative pupil size was also measured 6 months after the procedure using the CASIA 2. Patients were also asked about the level of discomfort due to the halo at 6 months postoperatively and responses were scored according to the answers "not at all" (0), "a little" (1), "somewhat" (2), "very much" (3), and "extreme" (4).

\section{Surgical Technique}

All surgical procedures were performed using femtosecond laser-assisted cataract surgery on the LenSx platform (Alcon Laboratories, Inc., Fort Worth, TX, USA) with a 5.0-mm capsulotomy and chop-and-cylinder fragmentation pattern. Emulsification and aspiration of the fragmented lens and aspiration of the cortex were performed using the Centurion $^{\circledR}$ Vision System (Alcon Laboratories, Inc., Fort Worth, TX, USA). In all cases, the IOLs were inserted in the bag.

\section{Data Analysis}

The Mann-Whitney $U$-test was used to compare halo size and intensity of the PanOptix and Symfony IOLs 1, 3, and 6 months after implantation. The changes in halo size and intensity with each IOL were analyzed between 1, 3, and 6 months after surgery using the Wilcoxon signed-rank test.

A post-hoc power analysis was performed to investigate the power of our tests from the given sample size. We investigated the correlations between halo size and age, pre and postoperative pupil size, and the actual value of postoperative refraction with each IOL. Additionally, the change in halo size with postoperative refraction correction 6 months postoperatively was analyzed. Furthermore, the relationship between 6-months postoperative halo size and the level of discomfort due to the halo was also analyzed. These relationships were analyzed for each IOL using the Spearman's rank-order correlation tests and $\mathrm{P}<0.05$ was considered statistically significant. All statistical analyses were performed using the Bell Curve 
for Excel software, version 3.20 (Social Survey Research Information Co., Ltd., Tokyo, Japan).

\section{Results}

The clinical characteristics of the 160 patients (80 in the PanOptix and 80 in the Symfony group) are shown in Table 1. The mean age, mean preoperative pupil size, and mean preoperative refraction were as follows: 74.1 \pm 5.7 years $(58-83), 3.26 \pm 0.3 \mathrm{~mm}(2.72-4.00)$, and -0.11 $\pm 1.57 \mathrm{D}(-4.25-3.00)$ in the Symfony group and 74.1 \pm 5.6 years (58-84), $3.23 \pm 0.28 \mathrm{~mm}$ (2.63-3.99), and -0.10 $\pm 1.58 \mathrm{D}(-3.50-2.50)$ in the PanOptix group, respectively. Women comprised $42 \%$ of the Symfony group and $43 \%$ of the PanOptix group. The mean postoperative pupil size and refraction were as follows: $3.01 \pm 0.28 \mathrm{~mm}(2.51$ $-3.71)$ and $-0.02 \pm 0.38 \mathrm{D}(-0.75-0.50)$ in the Symfony group and $3.00 \pm 0.26 \mathrm{~mm}(2.43-3.70)$ and $-0.02 \pm 0.36$ $\mathrm{D}(-0.75-0.50)$ in the PanOptix group, respectively.

The post-hoc power analysis showed that, for the medium effect size $\mathrm{d}=0.5$, the Mann-Whitney $\mathrm{U}$ and Wilcoxon signed-rank tests had very good power (88.2\% and 99.3\%, respectively) (Additional Files 1 and 2 , respectively).

Table I Patients' Clinical Characteristics

\begin{tabular}{|l|l|l|}
\hline & $\begin{array}{l}\text { Symfony } \\
(\mathbf{n = 8 0 )}\end{array}$ & $\begin{array}{l}\text { PanOptix } \\
(\mathbf{n = 8 0})\end{array}$ \\
\hline Age (years) & $74.1 \pm 5.7(58-83)$ & $74.1 \pm 5.6(58-84)$ \\
Sex (Female) & $42 \%$ & $43 \%$ \\
Preoperative photopic pupil & $3.26 \pm 0.3(2.72$ & $3.23 \pm 0.28(2.63$ \\
size (mm) & $-4.00)$ & $-3.99)$ \\
Preoperative refraction (D) & $-0.1 I \pm 1.57$ & $-0.10 \pm 1.58$ \\
& $(-4.25-3.00)$ & $(-3.50-2.50)$ \\
Postoperative photopic & $3.0 I \pm 0.28(2.5 \mathrm{I}$ & $3.00 \pm 0.26(2.43$ \\
pupil size (mm) & $-3.7 \mathrm{I})$ & $-3.70)$ \\
Postoperative refraction (D) & $-0.02 \pm 0.38$ & $-0.02 \pm 0.36$ \\
& $(-0.75-0.50)$ & $(-0.75-0.50)$ \\
\hline
\end{tabular}

Abbreviation: D, diopters.

\section{Size and Intensity of the Halo with PanOptix and Symfony}

Table 2 indicates the halo size with each IOL at 1, 3, and 6 months postoperatively. The halo size with PanOptix (4.60 $\pm 1.80,4.40 \pm 1.79,3.20 \pm 1.54)$ was significantly larger than that with Symfony $(3.73 \pm 2.03,3.46 \pm 1.84,2.90 \pm$ $1.87)$ at 1,3 , and $6(\mathrm{P}<0.05)$. With each $\mathrm{IOL}$, the halo size decreased significantly between 1 and 3 months $(\mathrm{P}<0.001)$ and between 3 and 6 months $(\mathrm{P}<0.001)$. The halo size decrease with each IOL was significantly larger between 3 and 6 months than between 1 and 3 months $(\mathrm{P}<0.001)$.

Table 3 shows the halo intensity in eachIOL at 1, 3 , and 6 months postoperatively. The halo intensity with Symfony $(4.28 \pm 1.79,4.09 \pm 1.74,3.41 \pm 1.69)$ was significantly higher than that with PanOptix $(3.93 \pm 1.51$, $3.71 \pm 1.34,2.50 \pm 1.19)(\mathrm{P}<0.05)$. With each IOL, the halo intensity decreased significantly between 1 and 3 months $(\mathrm{P}<0.001)$ and between 3 and 6 months $(\mathrm{P}<0.001)$. With each IOL, the halo intensity was significantly lower between 3 and 6 months than between 1 and 3 months $(\mathrm{P}<0.001)$.

The $\mathrm{T} 1$ halo pattern (diffuse halo ring) was observed in $56.6 \%$, T2 (starburst type) was observed in $43.4 \%$, and T3 (distinct halo ring) was observed in $0 \%$ of patients in the Symfony group. The T1, T2, and T3 patterns were observed in $58.2 \%, 41.8 \%$, and $0 \%$ of patients in the PanOptix group, respectively. There were no significant differences between the types of halo with both IOLs $(\mathrm{P}>0.05)$.

\section{Relationship Between Halo Size and Age, Pre- and Postoperative Pupil Size, and Postoperative Refraction}

The preoperative photopic pupil size demonstrated a significantly positive correlation with halo size 6 months preoperatively in the Symfony $(\mathrm{Rho}=0.584 \mathrm{P}<0.0001)$ (Figure 1A) and PanOptix (Rho=0.679 $\mathrm{P}<0.0001$, $\mathrm{Rho}=0.591 \mathrm{P}<0.0001$ ) (Figure 1B) groups.

Table 2 Time Trends in Postoperative Halo Size at I, 3, and 6 Months

\begin{tabular}{|c|c|c|c|c|c|c|}
\hline \multirow[t]{2}{*}{ Lens } & \multicolumn{3}{|c|}{ Halo Size Post Operation } & \multirow[t]{2}{*}{$* P_{1 M-3 M}$} & \multirow[t]{2}{*}{$* P_{3 M-6 M}$} & \multirow[t]{2}{*}{$* P_{(I M-3 M)-(3 M-6 M)}$} \\
\hline & IM & $3 M$ & $6 M$ & & & \\
\hline Symfony & $3.73 \pm 2.03$ & $3.46 \pm 1.84$ & $2.90 \pm 1.87$ & $<0.001$ & $<0.001$ & $<0.001$ \\
\hline PanOptix & $4.60 \pm 1.80$ & $4.40 \pm 1.79$ & $3.20 \pm 1.54$ & $<0.001$ & $<0.001$ & $<0.001$ \\
\hline \multicolumn{2}{|c|}{ *Wilcoxon signed-rank test } & & & & & \\
\hline
\end{tabular}

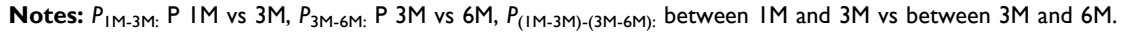

Abbreviations: IM, I month; $3 M, 3$ months; $6 \mathrm{M}, 6$ months. 
Table 3 Time Trends in Postoperative Halo Intensity at I, 3, and 6 Months

\begin{tabular}{|l|l|l|l|l|l|l|}
\hline \multirow{2}{*}{ Lens } & \multicolumn{2}{|l|}{ Halo Intensity Post Operation } & \multirow{2}{*}{$\boldsymbol{P}_{\text {IM-3M }}$} & \multirow{2}{*}{$\boldsymbol{P}_{\text {3M-6M }}$} & $\boldsymbol{P}_{\text {(IM-3M)-(3M-6M) }}$ \\
\cline { 2 - 5 } & IM & $3 \mathrm{M}$ & $6 \mathrm{M}$ & & \\
\hline $\begin{array}{l}\text { Symfony } \\
\text { PanOptix }\end{array}$ & $4.28 \pm 1.79$ & $4.09 \pm 1.74$ & $3.41 \pm 1.69$ & $<0.001$ & $<0.001$ & $<0.001$ \\
\hline
\end{tabular}

Notes: $P_{1 M-3 M}: P$ IM vs 3M, P3M-6M: $P$ 3M vs 6M, $P_{(I M-3 M)-(3 M-6 M)}$ : between IM and $3 M$ vs $3 M$ and $6 M$.

Abbreviations: IM, I month; 3M, 3 months; $6 \mathrm{M}, 6$ months.

The postoperative photopic pupil size demonstrated a significantly positive correlation with halo size 6 months postoperatively in the Symfony ( $R h o=0.589$ $\mathrm{P}<0.0001)$ (Figure 2A) and PanOptix (Rho $=0.690$ $\mathrm{P}<0.0001$ ) (Figure 2B) groups. Postoperative refraction showed a significant negative correlation with halo size in the Symfony ( $\mathrm{Rho}=-0.708 \quad \mathrm{P}<0.0001$ ) (Figure $3 \mathrm{~A}$ ) and PanOptix (Rho=-0.545 $\mathrm{P}<0.0001)$ (Figure 3B) groups 6 months postoperatively. Additionally, the higher the postoperative myopia was corrected, the more the halo size decreased in the Symfony (Rho= $-0.660 \mathrm{P}<0.0001$ ) (Figure $4 \mathrm{~A}$ ) and PanOptix (Rho= $-0.567 \mathrm{P}<0.0001) \quad($ Figure $4 \mathrm{~B})$ groups 6 months postoperatively.
A

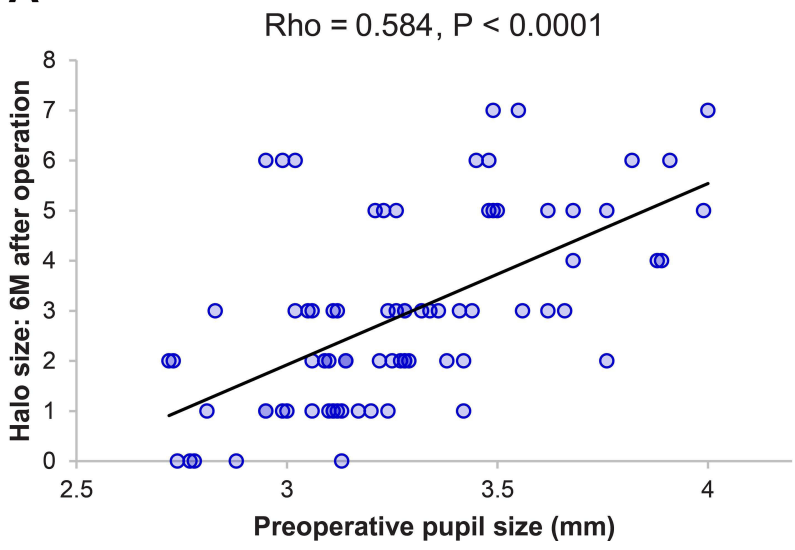

B

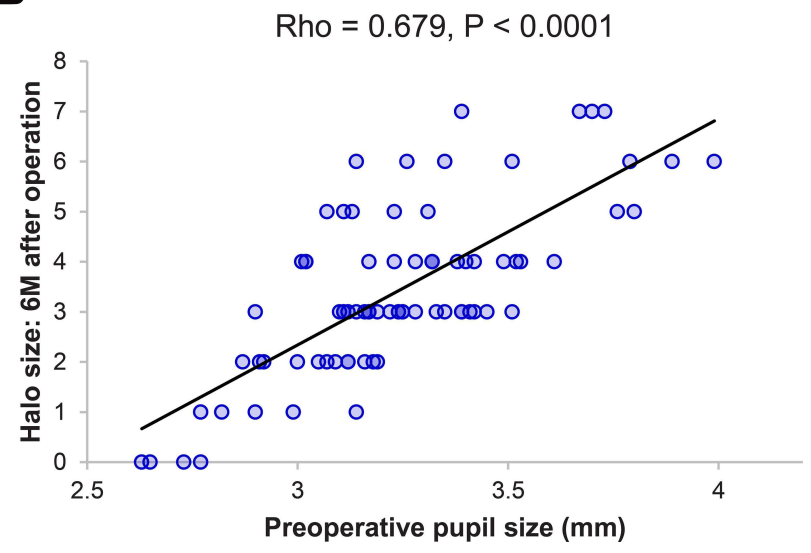

Figure I Correlation between the preoperative pupil size and halo size, measured 6 months postoperatively, with the Symfony (A) and PanOptix (B) intraocular lenses. Abbreviation: M, month.

A

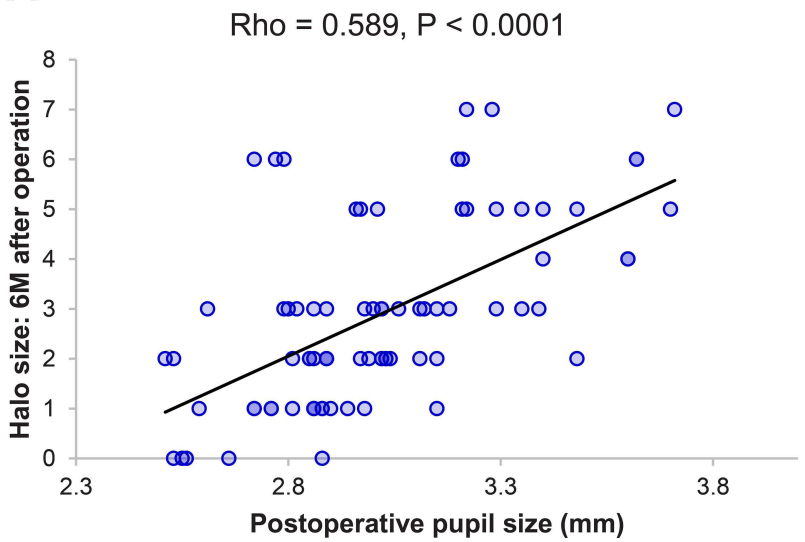

B

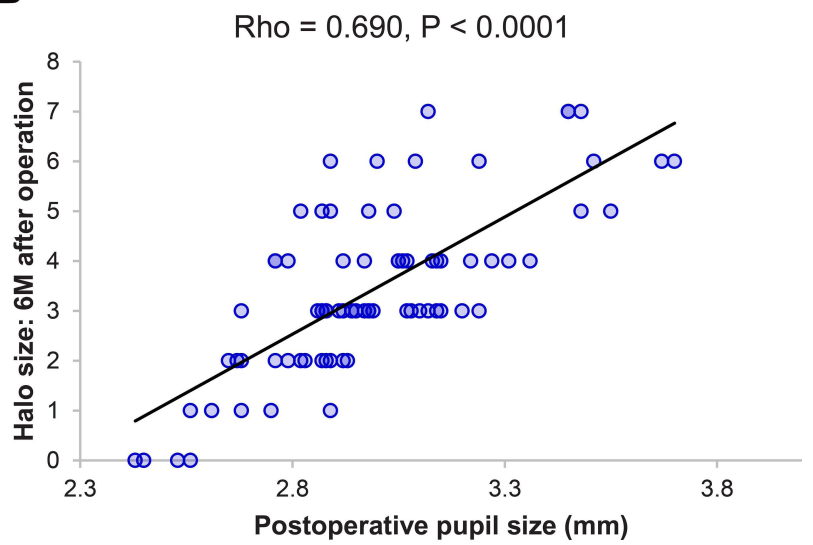

Figure 2 Correlation between the postoperative pupil size and halo size, measured 6 months postoperatively, with the Symfony (A) and PanOptix (B) intraocular lenses. Abbreviation: $M$, month. 

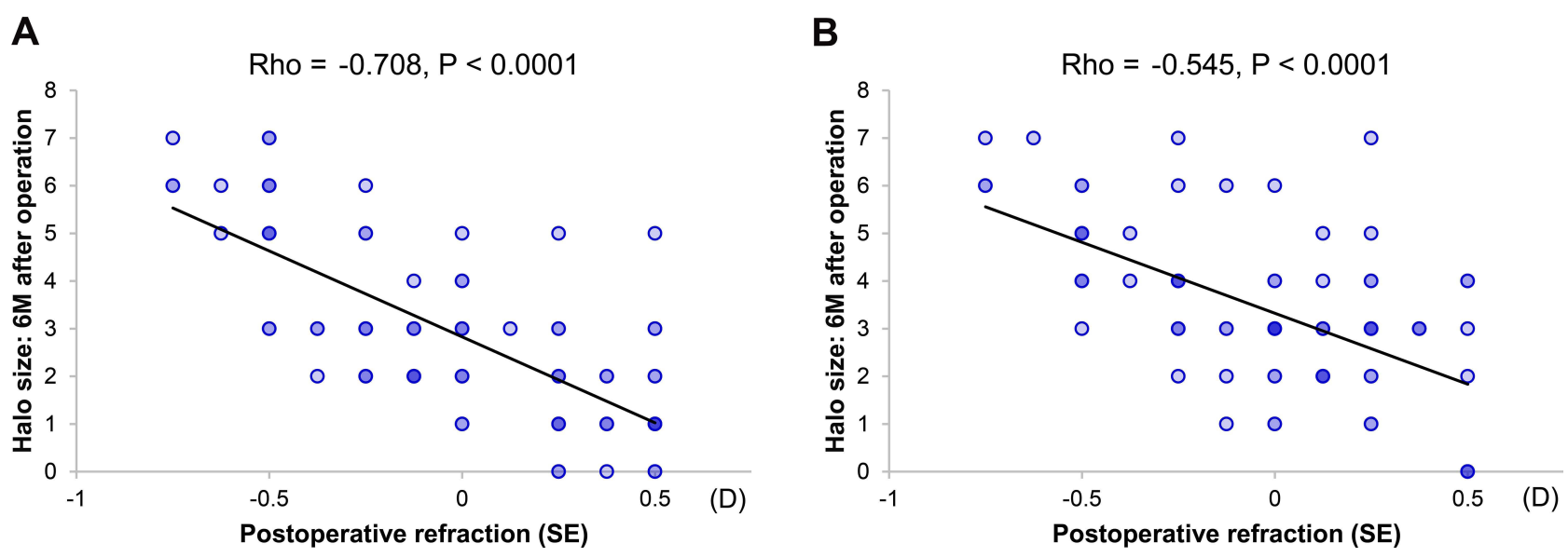

Figure 3 Correlation between postoperative refraction and halo size, measured 6 months postoperatively, with the Symfony (A) and PanOptix (B) intraocular lenses. Abbreviations: $M$, month; SE, spherical equivalent; D, diopters.
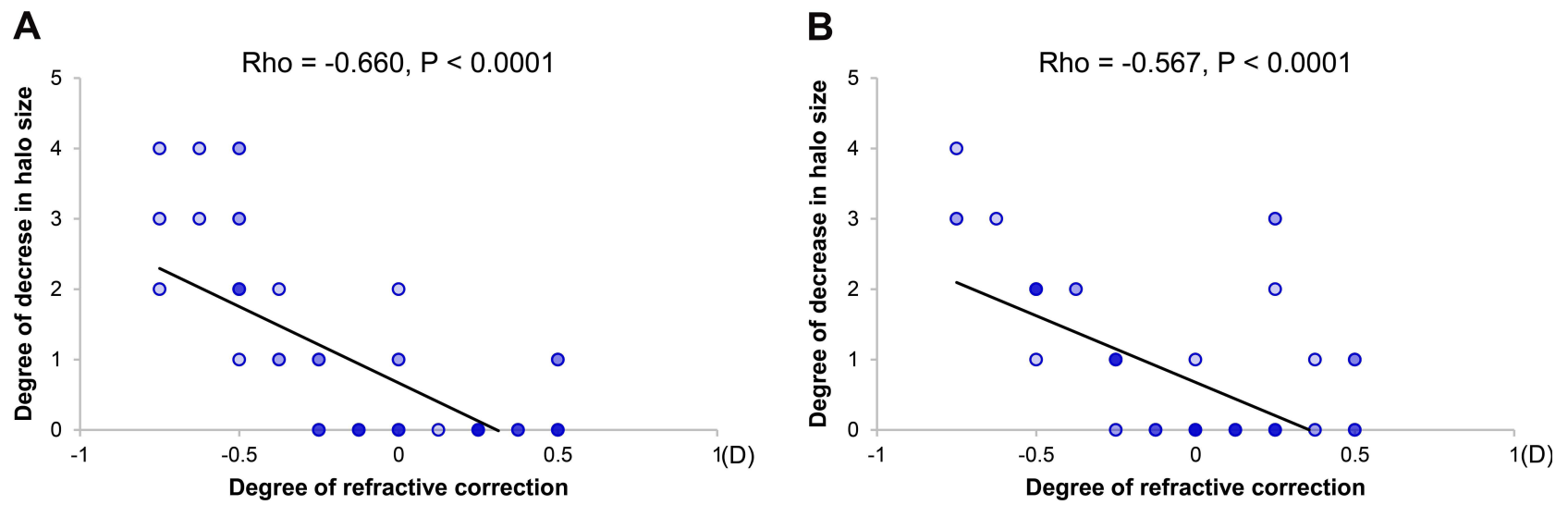

Figure 4 Correlation between the degree of refractive correction and the degree of the decrease in halo size, measured 6 months postoperatively, with the Symphony (A) and PanOptix (B) intraocular lenses.

Abbreviation: D, diopters.

The patients' age was negatively correlated with postoperative halo size 6 months postoperatively in both the Symfony and PanOptix groups. These correlations were significant in the Symfony $(\mathrm{Rho}=-0.589 \quad \mathrm{P}<0.0001)$ (Figure 5A) and PanOptix $(\mathrm{Rho}=-0.540 \quad \mathrm{P}<0.0001)$ (Figure 5B) groups.

\section{Relationships Between Age, Pre- and Postoperative Pupil Size, and Postoperative Refraction}

The pre- and postoperative pupil size exhibited significant positive correlations in the Symfony ( $\mathrm{Rho}=0.999 \mathrm{P}<0.0001$ ) (Figure 6A) and PanOptix (Rho=0.999 $\mathrm{P}<0.0001)$ (Figure 6B) groups. No significant correlation was observed between postoperative pupil size and postoperative refraction in the Symfony ( $\mathrm{Rho}=-0.030 \mathrm{P}=0.792)$ (Figure 7A) and
PanOptix (Rho $=-0.061 \mathrm{P}=0.558$ ) (Figure 7B) groups. Age and postoperative pupil size demonstrated significant negative correlations in the Symfony $(\mathrm{Rho}=-0.926 \mathrm{P}<0.0001)$ (Figure 8A) and PanOptix (Rho $=-0.827 \quad \mathrm{P}<0.0001$ ) (Figure $8 \mathrm{~B}$ ) groups. However, there was no significant correlation between age and postoperative refraction in the Symfony (Rho $=-0.030 \quad \mathrm{P}=0.792) \quad$ (Figure 9A) and PanOptix (Rho $=-0.061 \mathrm{P}=0.558$ ) (Figure 9B) groups.

\section{Level of Patient Discomfort Due to the Postoperative Halo}

The level of patient discomfort due to the postoperative halo overall and with each IOL type is shown in Figure 10A. There were no significant differences in the discomfort level between the IOLs ( $\mathrm{P}>0.05)$.

There was a significant positive correlation between halo size without refraction correction and the level of 

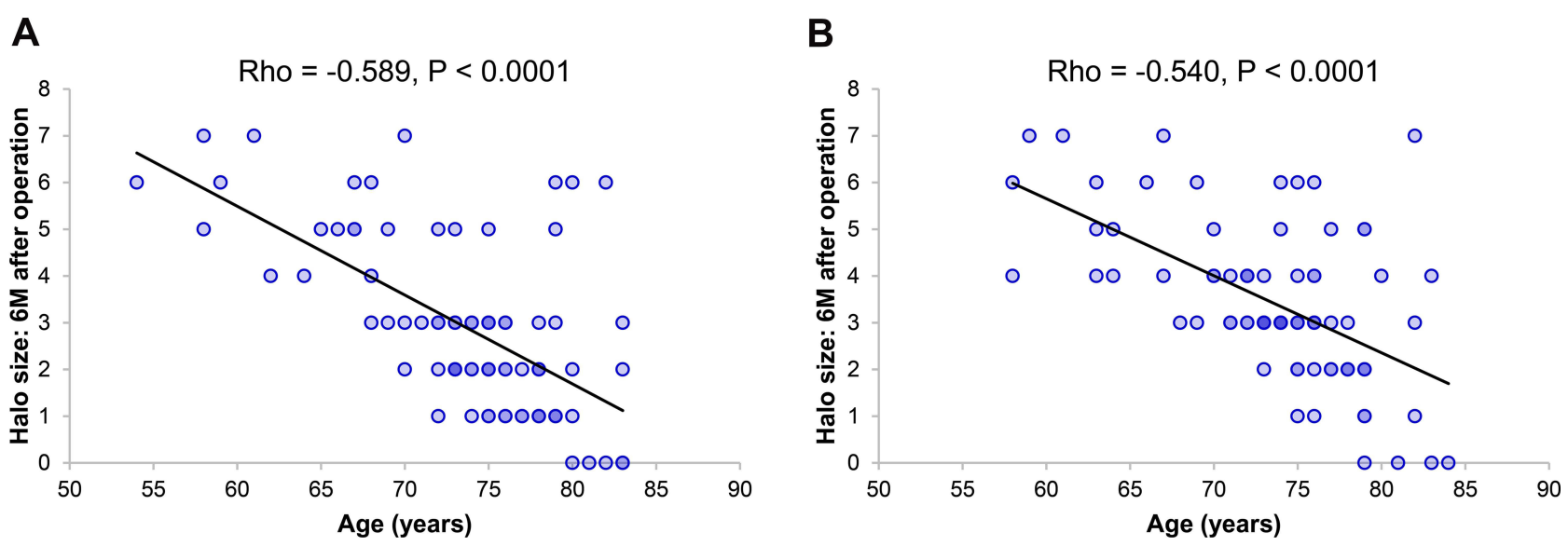

Figure 5 Correlation between age and halo size, measured 6 months postoperatively, with the Symfony (A) and PanOptix (B) intraocular lenses. Abbreviation: $\mathrm{M}$, month.

A

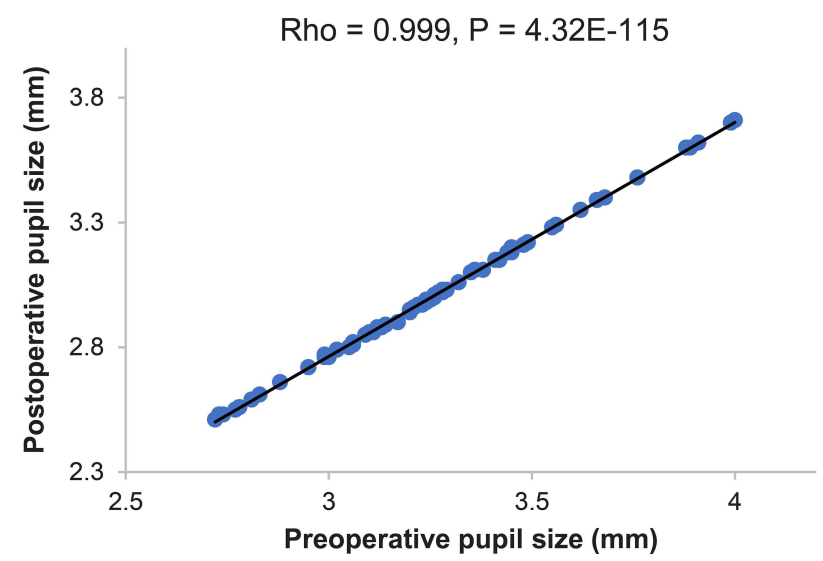

B

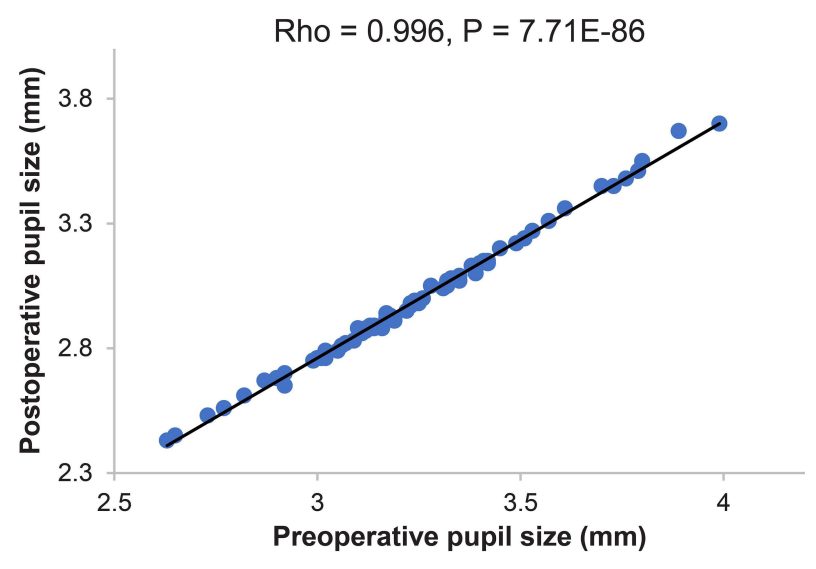

Figure 6 Correlation between preoperative and postoperative pupil size with the Symfony (A) and PanOptix (B) intraocular lenses.

A

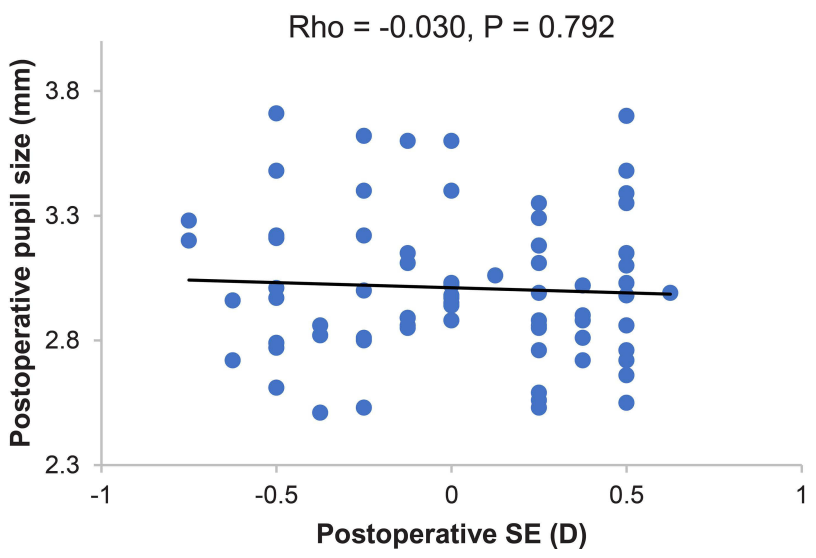

B

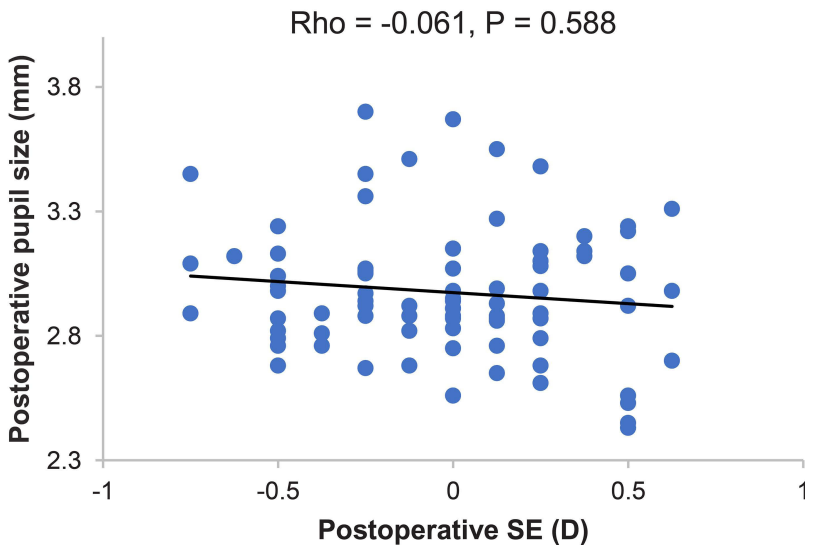

Figure 7 Correlation between postoperative pupil size and postoperative refraction with the Symfony (A) and PanOptix (B) intraocular lenses. Abbreviations: SE, spherical equivalent; D, diopters. 

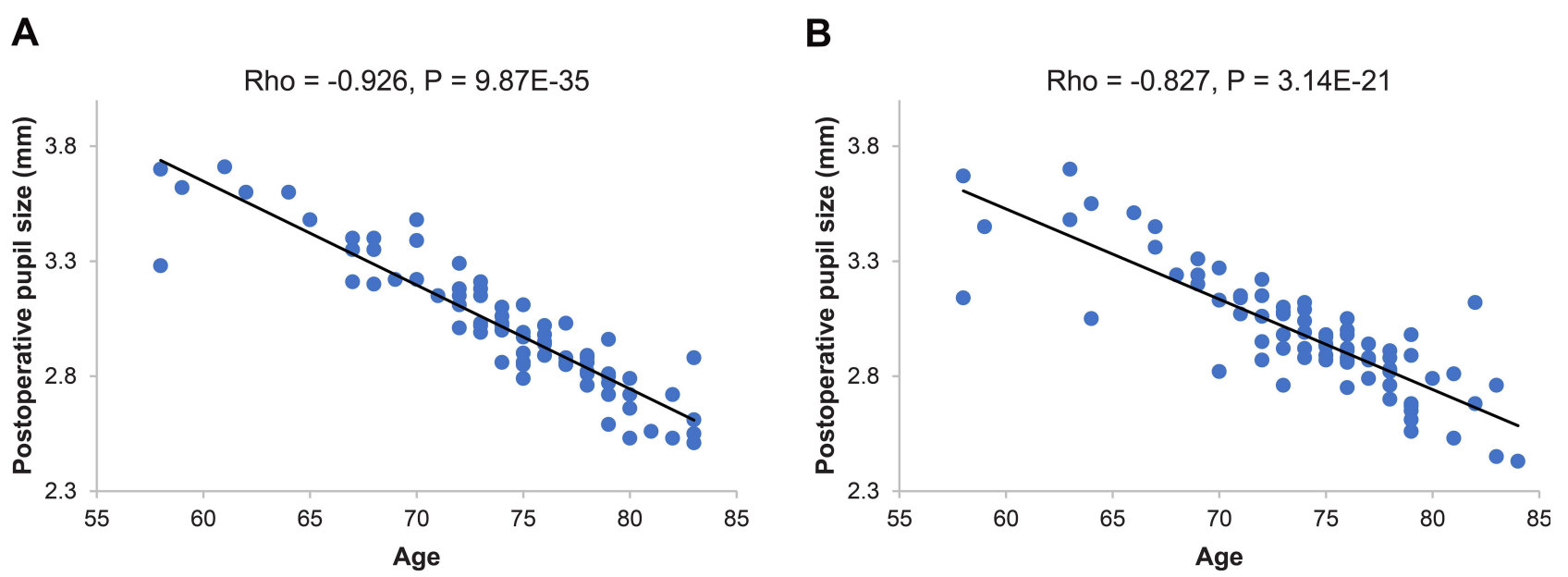

Figure 8 Correlation between age and postoperative pupil size with the Symfony $(\mathbf{A})$ and PanOptix (B) intraocular lenses.

A

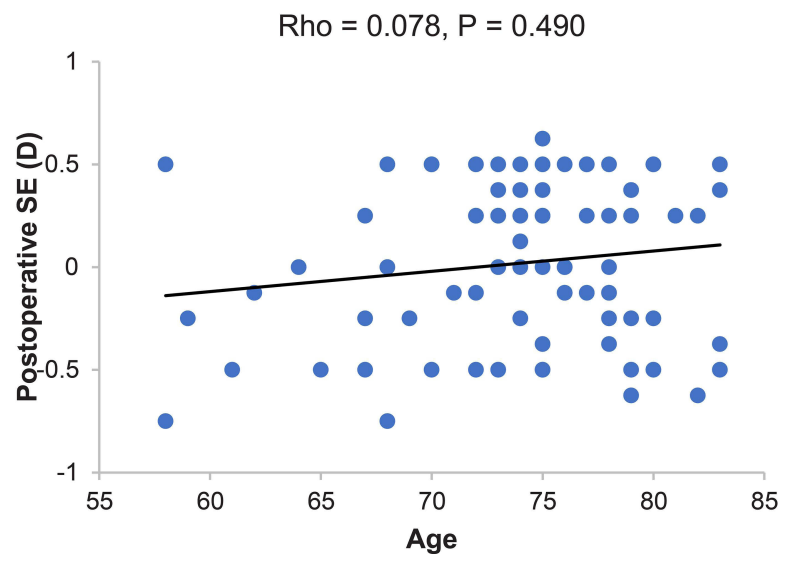

B

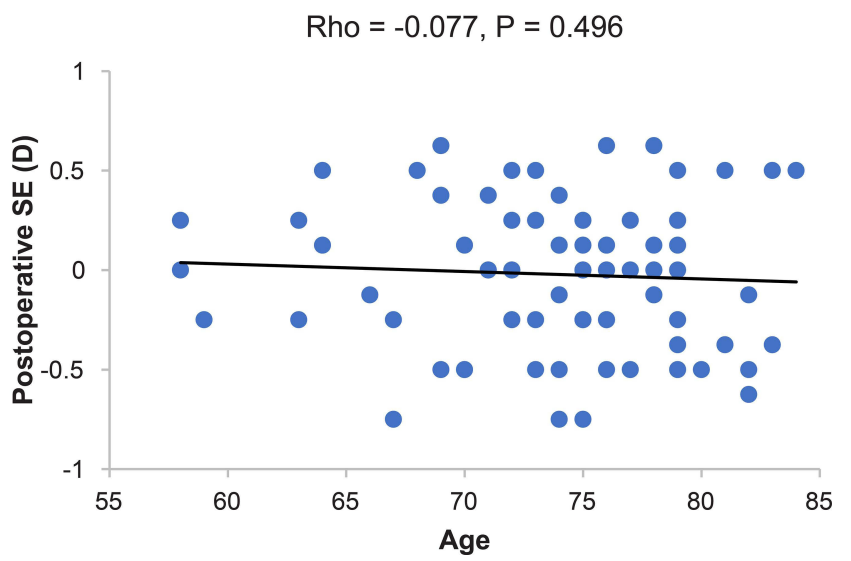

Figure 9 Correlation between age and postoperative refraction with the Symfony $(\mathbf{A})$ and PanOptix (B) intraocular lenses. Abbreviations: SE, spherical equivalent; D, diopters.

patient discomfort 6 months postoperatively in both the Symfony $(\mathrm{Rho}=0.852 \mathrm{P}<0.0001$ ) (Figure $10 \mathrm{~B}$ ) and PanOptix $(\mathrm{Rho}=0.847 \mathrm{P}<0.0001)$ groups (Figure 10C).

\section{Discussion}

This study focused on analyzing the relationship between the photic phenomena (halo) and the potential predictive factors of halo size and the time trend in halo size and intensity in eyes implanted with a trifocal or EDOF lens.

A halo is formed by the superimposition of 1 or more focus images produced by the different powers present in the optical zone of multifocal IOLs. Alba-Bueno et $\mathrm{al}^{21}$ conducted in vitro objective assessments of the halo and found that the size of the halo increases in proportion to the added power and that halo size negatively correlated with intensity. The results of our study correspond to their findings, although the halo was significantly larger with trifocal (PanOptix) than with EDOF (Symfony) IOLs, and vice versa for intensity. Moreover, it has also been reported that the subjective perception of halos is mitigated by the passage of time, ${ }^{6,22}$ probably due to neuroadaptation. ${ }^{13}$ In this study, halo size and intensity with both IOLs decreased at comparable levels in the observation period, which can be attributable to neuroadaptation. The decrease in halo size and intensity with each IOL was significantly greater between 3 and 6 months than between 1 and 3 months in our study. Thus, the results of the size and intensity of the halo in trifocal (PanOptix) and EDOF (Symfony) IOLs in our study supported the optical and physical theories of previous studies. ${ }^{6,21,22}$ Hence, patients should be notified of these tendencies, so that they can have a realistic 

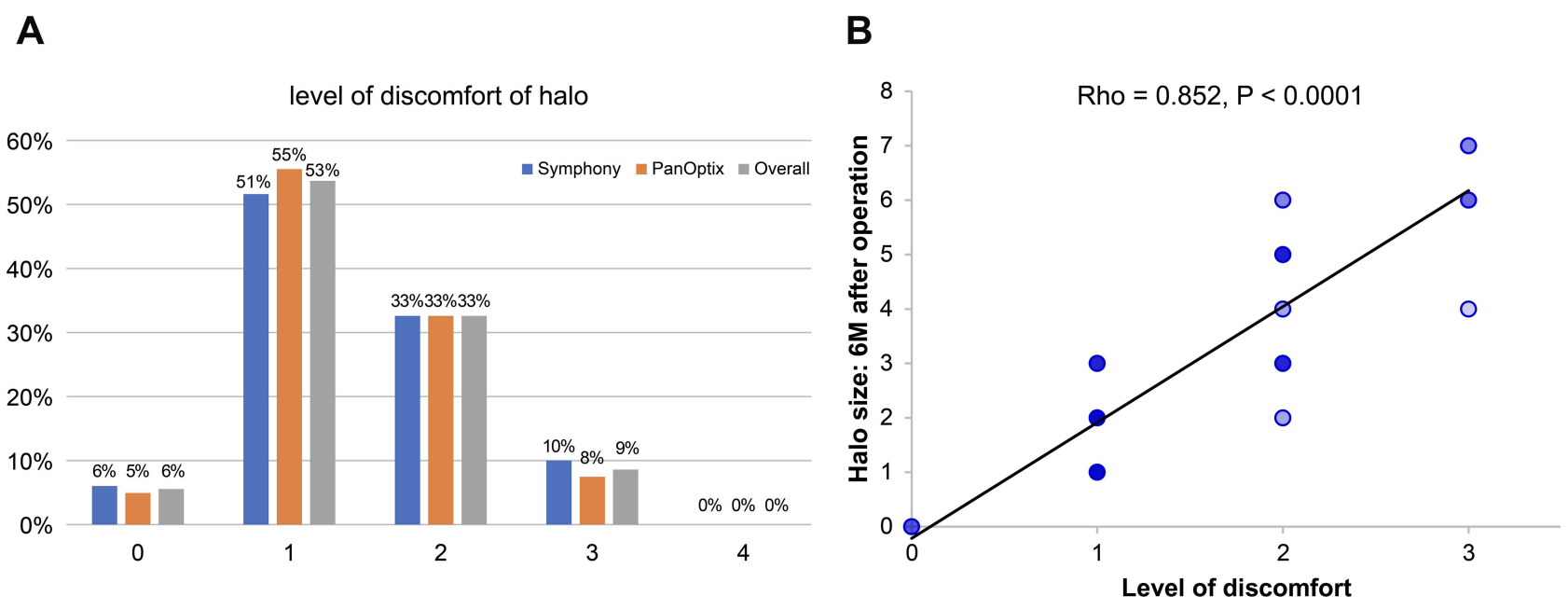

C

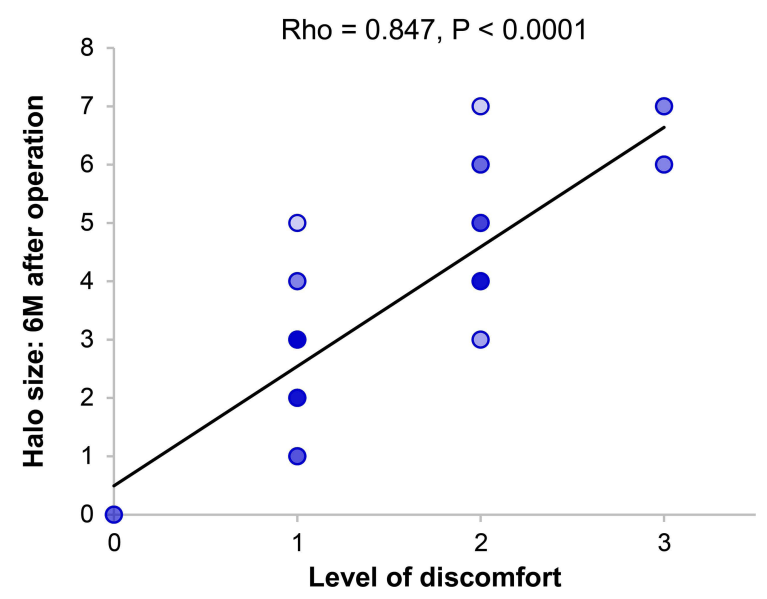

Figure 10 Level of discomfort due to the postoperative halo overall and with the Symfony and PanOptix intraocular lenses (A) 6 months postoperatively, where: $0=$ not at all, I = a little, 2 = somewhat, 3 = very much, 4 = extreme. Correlation between level of discomfort and halo size measured 6 months postoperatively in the Symfony (B) and PanOptix (C) groups.

expectation about the quality of postoperative vision in multifocal IOLs.

The prediction of the degree of postoperative halos using pre-operative variables aids the selection of multifocal IOL type and provides patients with a more personalized explanation of the possibility of halo incidence before implantation. This notion was the motivation underlying our aim to analyze the relationship between halo size and pre-operative variables.

Prakash et $\mathrm{al}^{15}$ demonstrated that halos significantly depend on the misalignment between the visual and pupillary axes (kappa angle) in multifocal IOLs. Tchah et $\mathrm{al}^{14}$ reported the importance of the large pupil center shift and kappa angle on the incidence of halos. The abovementioned factors are postoperative factors that influence halo occurrence. Our objective was to explore preoperative factors that could predict halos. Some studies have discussed the relationship between preoperative pupil size and postoperative halos in laser vision correction, such as laser in situ keratomileusis. ${ }^{23-25}$ However, only a few studies have investigated the relationship between preoperative pupil size and halos in multifocal IOLs. Pieh et $\mathrm{al}^{26}$ reported that halo size depends on pupil diameter; however, their study investigated refractive multifocal IOLs and did not include diffractive multifocal IOLs. Vega et $\mathrm{al}^{12}$ showed that the halo size increases with an increase in pupil size in 4 different types of diffractive multifocal IOLs, and that the halo is the largest when the pupil size is $4.5 \mathrm{~mm}$. Nonetheless, their research was conducted in vitro and not in vivo. Therefore, to the best of our knowledge, this is the first in vivo study to report the relationship between halo and pupil size in diffractive multifocal IOLs. Pre- and postoperative pupil size exhibited a significant positive correlation with halo size with 
both IOLs in our study. It is known that halo formation results from the superimposition of 1 or more out-of-focus images (derived from the different powers existing in the optical zone of the multifocal IOL) on a focused image, resultantly larger pupils are responsible for wider out-offocus images. This image is displayed on the retina and can contribute to halo size. Moreover, in our study, pupil size decreased after surgery. According to Kanellopoulos et $\mathrm{al}^{27}$ both horizontal and vertical pupil diameters decrease after cataract surgery. In our study, preoperative pupil size, which decreased after surgery, exhibited a strong, statistically significant positive correlation with postoperative pupil size. These results indicate that the preoperative pupil size may be used to predict halo size in multifocal IOLs.

Theoretically, all the light rays from a point source are on-axis in a perfectly focused eye (plano). In a myopic eye, however, light rays are focused in front of the retina and diffuse when they reach the retina. These off-axis light rays can affect the degree of halo formation. ${ }^{21}$

However, in clinical settings, patients with preoperative myopia sometimes complain of uncorrected near sightedness after multifocal IOL implantation when the postoperative target refraction is plano. One study reported that slight myopia can be suitable for postoperative target refraction to improve uncorrected near sightedness, and patient satisfaction can improve with trifocal IOLs. ${ }^{28}$ Therefore, we sometimes use very slight myopia as the postoperative target refraction in order to improve postoperative uncorrected near sightedness. In our study, the postoperative refraction demonstrated a significant negative correlation with halo size, and this result implies a positive association between the degree of myopia in postoperative refraction and halo size. Moreover, in this study, halo size decreased with refractive correction in postoperative myopic eyes, and the higher the myopia was corrected, the more the halo size decreased with each IOL. This tendency was not seen in postoperative hyperopic eyes. These results indicate that target refraction can be a predictive factor of postoperative halo size. Therefore, when myopia is the target, it is advisable to inform patients about possible postoperative halos in the preoperative consultation.

Regarding the relationship between postoperative pupil size and postoperative refraction, the results showed that postoperative pupil size did not exhibit a significant correlation with postoperative refraction. Therefore, it can be considered that postoperative refraction and postoperative pupil size were significantly and independently correlated with halo size.

This study also demonstrated that age could be one of the predictive factors for halo size. First, there was a significantly strong negative correlation between age and postoperative pupil size. Other studies have also reported a similar negative correlation between age and pupil size. ${ }^{29,30}$ Second, age showed a significant negative correlation with halo size. These findings show that age and pupil size are probably negatively correlated. Therefore, we can assume that age may be a predictive factor for halo size.

Concerning the level of discomfort due to the postoperative halo, there was no significant difference between the two IOLs. With each IOL, the larger the halo size, the more discomfort the patients experienced. These are also important results to increase postoperative patient satisfaction.

The main strength of this study is that it may pioneer research into the predictive preoperative variables of postoperative halo size. This study has some limitations. Although the characteristics of the halo in the different types of IOLs are also attributable to the lens design, such as apodization or non-apodization, and sphericity or asphericity, ${ }^{15,23}$ those factors were beyond the scope of this study. Additionally, there is a drawback in the computer-based halo simulator, namely that it is likely for patients to indicate the halo size and intensity derived from the memory when they were driving; therefore, there is a possibility for the patients to over- or underestimate the magnitude of the halos. Another limitation of this study is that the influence of the shape of the eye was not analyzed. Therefore, in future studies, the relationship between the shape of the eye and halo size should be investigated to verify if the postoperative refraction is an independent factor in producing halos. Moreover, as we age, retinal sensitivity and media changes (vitreous and cornea) may also affect halos, and these were not investigated in this study. Furthermore, the power of our tests was very good for a medium size effect $(d=0.5)$; however, it was low for a small size effect $(d=0.2)$. Therefore, the findings of these tests may be considered strongly indicative rather than conclusive. Glare is also a common photic phenomenon with multifocal IOLs. However, in this study we focused on halos since glare cannot be clearly differentiated from halos, and previous studies have more commonly investigated halos rather than glare. ${ }^{31-33}$ 


\section{Conclusions}

This study showed that the change in halo size and intensity of the trifocal (PanOptix) and EDOF (Symfony) IOLs over time should be taken into consideration during treatment planning. Age, target refraction, and preoperative pupil size may be predictive factors for halo size, although further investigation is required into the mechanism underlying these associations. An in-depth understanding of these correlations may be useful for managing patient expectations of postoperative quality of vision and possibly improve postoperative patient satisfaction.

The management of target refraction may be especially crucial for minimizing halo size, since this is the only adjustable preoperative factor.

\section{Funding}

There is no funding to report.

\section{Disclosure}

The authors declare that they have no competing interests.

\section{References}

1. Zhao G, Zhang J, Zhou Y, Hu L, Che C, Jiang N. Visual function after monocular implantation of apodized diffractive multifocal or single-piece monofocal intraocular lens: randomized prospective comparison. J Cataract Refract Surg. 2010;36:282-285. doi:10.1016/ j.jcrs.2009.08.037

2. Nijkamp MD, Dolders MG, de Brabander J, van den Borne B, Hendrikse F, Nuijts RM. Effectiveness of multifocal intraocular lenses to correct presbyopia after cataract surgery: a randomized controlled trial. Ophthalmology. 2004;111:1832-1839. doi:10.1016/j.ophtha.20 04.05.023

3. de Vries NE, Webers CAB, Touwslager WRH, et al. Dissatisfaction after implantation of multifocal intraocular lenses. $J$ Cataract Refract Surg. 2011;37:859-865. doi:10.1016/j.jcrs.2010.11.032

4. Mencucci R, Favuzza E, Caporossi O, Savastano A, Rizzo S. Comparative analysis of visual outcomes reading skills, contrast sensitivity, and patient satisfaction with two models of trifocal diffractive intraocular lenses and an extended range of vision intraocular lens. Graefes Arch Clin Exp Ophthalmol. 2018;256:1913-1922. doi:10.10 07/s00417-018-4052-3

5. de Silva SR, Evans JR, Kirthi V, Ziaei M, Leyland M. Multifocal versus monofocal intraocular lenses after cataract extraction. Cochrane Database Syst Rev. 2016;12:CD003169.

6. Kamiya K, Hayashi K, Shimizu K, et al. Multifocal intraocular lens explantation: a case series of 50 eyes. Am $J$ Ophthalmol. 2014;158:215-220. doi:10.1016/j.ajo.2014.04.010

7. Ruiz-Mesa R, Abengózar-Vela A, Ruiz-Santos M. A comparative study of the visual outcomes between a new trifocal and an extended depth of focus intraocular lens. Eur J Ophthalmol. 2018;28:182-187. doi:10.5301/ejo.5001029

8. Skiadaresi E, McAlinden C, Pesudovs K, Polizzi S, Khadka J, Ravalico G. Subjective quality of vision before and after cataract surgery. Arch Ophthalmol. 2012;130:1377-1382. doi:10.1001/archophthalmol.2012. 1603
9. Wolffe M, Landry RJ, Alpar JJ. Identification of the source of permanent glare from a three-piece IOL. Eye. 2007;21:1078-1082. doi:10.1038/sj.eye. 6702539

10. Alba BF, Vega F, Millán MS. Halos and multifocal intraocular lenses: origin and interpretation. Arch Soc Esp Oftalmol. 2014;89:397-404. doi:10.1016/j.oftal.2014.01.002

11. Ellis MF. Sharp-edged intraocular lens design as a cause of permanent glare. J Cataract Refract Surg. 2001;27:1061-1064. doi:10. 1016/S0886-3350(00)00856-7

12. Vega F, Alba-Bueno F, Millán MS, Varón C, Gil MA, Buil JA. Halo and through-focus performance of four diffractive multifocal intraocular lenses. Invest Ophthalmol Vis Sci. 2015;56:3967-3975. doi:10. 1167/iovs. $15-16600$

13. Sachdev GS, Sachdev M. Optimizing outcomes with multifocal intraocular lenses. Indian J Ophthalmol. 2017;65:1294-1300. doi:10. 4103/ijo.IJO_1072_17

14. Tchah H, Nam K, Yoo A. Predictive factors for photic phenomena after refractive, rotationally asymmetric, multifocal intraocular lens implantation. Int J Ophthalmol. 2017;10:241-245.

15. Prakash G, Prakash RD, Agarwal A, Kumar DA, Agarwal A, Jacob S. Predictive factor and kappa angle analysis for visual satisfactions in patients with multifocal IOL implantation. Eye. 2011;25:1187-1193. doi:10.1038/eye.2011.150

16. Pop M, Payette Y. Risk factors for night vision complaints after LASIK for myopia. Ophthalmology. 2004;111:3-10. doi:10.1016/j. ophtha.2003.09.022

17. Carson D, Xu Z, Alexander E, Choi M, Zhao Z, Hong X. Optical bench performance of 3 trifocal intraocular lenses. $J$ Cataract Refract Surg. 2016;42:1361-1367. doi:10.1016/j.jcrs.2016.06.036

18. Lee S, Choi M, Xu Z, Zhao Z, Alexander E, Liu Y. Optical bench performance of a novel trifocal intraocular lens compared with a multifocal intraocular lens. Clin Ophthalmol. 2016;10:1031-1038. doi:10.2147/OPTH.S106646

19. Cochener B, Concerto Study Group. Clinical outcomes of a new extended range of vision intraocular lens: international Multicenter Concerto Study. J Cataract Refract Surg. 2016;42:1268-1275. doi:10.1016/j.jcrs.2016.06.033

20. Emery JM, Little JH. Phacoemulsification and Aspiration of Cataracts: Surgical Techniques, Complications and Results. St Louis: Mosby; 1979:45-48.

21. Alba-Bueno F, Garzón N, Vega F, Poyales F, Millán MS. Patientperceived and laboratory-measured halos associated with diffractive bifocal and trifocal intraocular lenses. Curr Eye Res. 2018;43:35-42. doi:10.1080/02713683.2017.1379541

22. Liu X, Xie L, Huang Y. Comparison of the visual performance after implantation of bifocal and trifocal intraocular lenses having an identical platform. J Refract Surg. 2018;34:273-280. doi:10.3928/ 1081597X-20180214-01

23. Schallhorn SC, Kaupp SE, Tanzer DJ, Tidwell J, Laurent J, Bourque LB. Pupil size and quality of vision after LASIK. Ophthalmology. 2003;110:1606-1614. doi:10.1016/S0161-6420(03) 00494-9

24. Schallhorn S, Brown M, Venter J, Hettinger K, Hannan S. The role of the mesopic pupil on patient reported outcomes in young patients with myopia 1 month after wavefront-guided LASIK. J Refract Surg. 2014;30:159-165. doi:10.3928/1081597X-20140217-02

25. Myung D, Schallhorn S, Manche EE. Pupil size and LASIK: a review. J Refract Surg. 2013;29:734-741. doi:10.3928/1081597X20131021-02

26. Pieh S, Lackner B, Hanselmayer G, et al. Halo size under distance and near conditions in refractive multifocal intraocular lenses. $\mathrm{Br}$ J Ophthalmol. 2001;85:816-821. doi:10.1136/bjo.85.7.816

27. Kanellopoulos JA, Asimellis G. Clear-cornea cataract surgery: pupil size and shape changes, along with anterior chamber volume and depth changes. A Scheimpflug imaging study. Clin Ophthalmol. 2014;8:2141-2150. doi:10.2147/OPTH.S68370 
28. Hayashi K, Sato T, Igarashi C, Yoshida M. Effect of spherical equivalent error on visual acuity at various distances in eyes with a trifocal intraocular lens. J Refract Surg. 2019;35:274-279. doi:10.3928/1081597X-20190404-01

29. Guillon M, Dumbleton K, Theodoratos P, Gobbe M, Wooley CB, Moody K. The effects of age, refractive status, and luminance on pupil size. Optom Vis Sci. 2016;93:1093-1100. doi:10.1097/ OPX.0000000000000893

30. Netto VM, Ambrósio R, Wilson ES. Pupil size in refractive surgery candidates. J Refract Surg. 2004;20:337-342. doi:10.3928/1081597X-20040701-06
31. Vega F, Alba-Bueno F, Mill'an MS, et al. Halo and through-focus performance of four diffractive multifocal intraocular lenses. Invest Ophthalmol Vis Sci. 2015;56:3967-3975.

32. Perez Carrasco M, Álvarez-rementería L, Hurtado-Ceña F, et al. Halo size after implantation of a multifocal intraocular lens. Acta Ophthalmol. 2013;91. doi:10.1111/j.1755-3768.2013.4458.x

33. Pieh S, Lackner B, Hanselmayer G, et al. Halo size under distance and near conditions in refractive multifocal intraocular lenses. $\mathrm{Br}$ J Ophthalmol. 2001;85:816-821.

\section{Publish your work in this journal}

Clinical Ophthalmology is an international, peer-reviewed journal covering all subspecialties within ophthalmology. Key topics include: Optometry; Visual science; Pharmacology and drug therapy in eye diseases; Basic Sciences; Primary and Secondary eye care; Patient Safety and Quality of Care Improvements. This journal is indexed on PubMed

Submit your manuscript here: https://www.dovepress.com/clinical-ophthalmology-journal
Central and CAS, and is the official journal of The Society of Clinical Ophthalmology (SCO). The manuscript management system is completely online and includes a very quick and fair peer-review system, which is all easy to use. Visit http://www.dovepress.com/ testimonials.php to read real quotes from published authors. 